\title{
Inflammatory bowel disease and Parkinson's disease: common pathophysiological links
}

Ho-Su Lee ${ }^{1,2^{*}}$, Evy Lobbestael ${ }^{3^{*}}$, Séverine Vermeire ${ }^{4}$, João Sabino ${ }^{4}$, and Isabelle Cleynen ${ }^{1,{ }^{*}, \#}$

${ }^{1}$ Laboratory of Complex Genetics, Department of Human Genetics, KU Leuven, 3000 Leuven, Belgium

${ }^{2}$ Department of Biochemistry and Molecular Biology, University of Ulsan College of Medicine, 05505 Seoul, Korea

${ }^{3}$ Laboratory for Neurobiology and Gene Therapy, KU Leuven, 3000 Leuven, Belgium

${ }^{4}$ Department of Gastroenterology and Hepatology, University Hospitals Leuven, 3000 Leuven, Belgium

*These authors contributed equally to this work.

${ }^{\#}$ Corresponding author: isabelle.cleynen@kuleuven.be

Keywords Inflammatory bowel disease; Parkinson's disease; Pleiotropy; LRRK2.

\section{Word count 5418}

Funding HSL was supported by a National Research Foundation of Korea (NRF) MRC grant funded by the Korean government (MSIT) (2018R1A5A2020732). EL is supported by the Research Foundation Flanders (FWO) (GOE1917N) and Michael J. Fox Foundation (17194).

Competing interests SV reports receiving speaker's fees from AbbVie, Takeda, Genentech/Roche, Pfizer Inc, and Janssen; receiving consultancy fees from AbbVie, MSD, Takeda, Ferring, Genentech/Roche, Shire, Pfizer Inc, Galapagos, Mundipharma, Hospira, Celgene, Second Genome, Progenity, Lilly, Arena, Gilead and Janssen; receiving financial support for research from MSD, Abbvie, Janssen and Pfizer. JS reports receiving speaker's fees from Nestle Health Sciences, Abbvie, and Takeda; receiving consultancy fees from Janssen. 


\begin{abstract}
Inflammatory bowel disease and Parkinson's disease are chronic progressive disorders that mainly affect different organs: the gut and brain, respectively. Accumulating evidence has suggested a bidirectional link between gastrointestinal inflammation and neurodegeneration, in accordance with the concept of the 'gut-brain axis'. Moreover, recent population-based studies have shown that inflammatory bowel disease might increase the risk of Parkinson's disease. Although the precise mechanisms underlying gut-brain interactions remain elusive, some of the latest findings have begun to explain the link. Several genetic loci are shared between both disorders with a similar direction of effect on the risk of both diseases. The most interesting example is $L R R K 2$ (leucine-rich repeat kinase 2 ), initially identified as a causal gene in Parkinson's disease, and recently also implicated in Crohn's disease. In this review, we highlight recent findings on the link between these seemingly unrelated diseases with shared genetic susceptibility. We discuss supporting and conflicting data obtained from epidemiologic and genetic studies along with remaining questions and concerns. In addition, we discuss possible biological links including the gut-brain axis, microbiota, autoimmunity, mitochondrial function, and autophagy.
\end{abstract}




\section{Key messages}

- A bidirectional link between gastrointestinal inflammation and neurodegeneration in the pathogenesis of Parkinson's disease (PD), in accordance with the idea of the 'gutbrain axis', has recently emerged.

- Population-based data reveal that patients with inflammatory bowel disease (IBD) are at higher risk of subsequently developing PD.

- The genetic overlap between IBD and PD suggests that shared disease mechanisms underlie these disparate disorders.

- Controlling the disease activity of IBD may reduce the risk of PD, potentially owing to a reduction in gut inflammation.

- Future therapeutic strategies could target the commonalities in the underlying biology to increase the resilience of the intestinal barrier and thus delay or prevent the onset of PD. 


\section{Box 1. Unanswered questions/concerns}

- Epidemiological questions

- Do patients with PD have a reduced risk of developing IBD?

- Do IBD patients with age at onset above 60-65 years have a higher risk of PD than IBD patients with younger age at onset?

- What triggers PD in patients with IBD?

- Do environmental factors (e.g., diet and smoking) play a role in the link between IBD and PD?

- Limitations of previous studies:

$\circ$ Is there an effect of surveillance bias?

- How can we accurately exclude secondary or atypical parkinsonism?

- Genetic questions

- Are some patients with PD genetically predisposed to intestinal inflammation?

- Which specific genetic predisposition explains the co-occurrence of IBD and PD?

- Is there a difference in the pleiotropic effects between the idiopathic and monogenic forms of IBD and PD?

- Limitations of previous studies:

- Low penetrance of shared genetic variants

- Preventing PD in patients with IBD

- Can we predict PD risk among patients with IBD?

- Identification of risk factors associated with prodromal phases of PD

- Does successful disease control alter the risk of co-occurrence?

- What are the effects of medication used to treat IBD on the risk of PD?

- What are the consequences of bowel resection on the risk of PD?

- Therapeutic interventions

- What is the role of immune-modulating agents in the general management of PD?

- Can modulation of the gut microbiota (e.g., fecal microbial transplantation) play a therapeutic role in prevention or management of PD? 


\section{Introduction}

Inflammatory bowel disease (IBD) and Parkinson's disease (PD) are both chronic progressive diseases resulting from a complex etiology. ${ }^{1-3}$ Both diseases differ in terms of age of onset, major suspected cause, main affected organ, and clinical symptoms (Table 1). IBD, which includes Crohn's disease (CD) and ulcerative colitis (UC), is a chronic condition of the gut and is accompanied by an impaired innate mucosal immune function, usually in young individuals. A histological feature of $C D$ are noncaseating granulomas, and cryptitis and/or crypt abscesses are common findings in UC. ${ }^{12}$ By contrast, PD is a neurodegenerative disorder of the aging brain, resulting in gradual worsening of movement including muscle rigidity, tremor, slowness of movement, and impaired coordination. PD is characterized by progressive loss of dopaminergic neurons in the substantia nigra and the presence of Lewy body pathology. Lewy bodies are abnormal aggregates of misfolded protein, mainly $\alpha$-synuclein ${ }^{4}$, which are tightly associated with neuronal dysfunction and degeneration.

The gut-brain axis involves bidirectional communication between the gut and brain, ${ }^{6}$ linking the two organs and also these seemingly unrelated diseases. Accumulating evidence has suggested that chronic intestinal inflammation in IBD can promote the development of PD. Indeed, recent population-based studies have shown that IBD patients are at higher risk of developing PD later in life. ${ }^{7-11}$ In addition, multiple studies support the idea of genetic pleiotropy, in which one gene influences two or more disparate phenotypic traits, in IBD and PD. ${ }^{12}$ Accordingly, research interest in the association between IBD and PD has risen steadily (Figure 1). In this review, we highlight epidemiologic and genetic studies that support a link between IBD and PD (Figure 2). We discuss why and how gastrointestinal inflammation, such as that seen in IBD, is believed to contribute to the early phases of PD pathogenesis, and provide the latest biological findings that may link the two diseases. Potential clinical implementation and therapeutic approaches are described where applicable.

\section{Epidemiological evidence linking IBD and PD}

Several epidemiological studies have sought to determine whether IBD increases the risk of PD (Table 2). To date, five population-based cohort studies have reported that the risk of PD is increased in IBD patients. ${ }^{7-11}$ Specifically, IBD patients are at a $20-90 \%$ higher risk of developing PD than individuals without IBD. However, the incidence of IBD among PD patients remains a topic of considerable debate. A US study using Medicare data showed that PD 
patients had a $15 \%$ lower risk of both CD and UC than non-PD controls. ${ }^{13}$ Hence, despite the elevated risk of PD among IBD patients, IBD risk does not seem to be elevated among PD patients. This suggests that the role of neurodegeneration in the pathogenesis of IBD is less important than the role of gut inflammation in the pathogenesis of PD. However, this discrepancy might also be related to the gap in peak incidence age between the two diseases. Indeed, IBD is usually diagnosed during early adulthood, while PD is rare before 50 years of age. ${ }^{3}$ In addition, typical symptoms of IBD (e.g. loose stool) could partially be masked in PD since a delay in gastric emptying and constipation are common in PD patients. ${ }^{14}$

Although this population-based research has provided important new insights into the link between PD and IBD, caution is warranted when interpreting the data. Since these data were initially collected for non-statistical reasons, the limitations of studies using administrative data include 1) surveillance bias, 2) the skewed age of the enrolled populations, 3) identifiers of disease (e.g., International Classification of Disease [ICD] version 9 versus ICD-10), and 4) confounding factors (e.g., medication, disease activity, history of bowel resection, and smoking). Surveillance bias, also known as detection bias, can occur if some IBD patients are followed up more closely than others. Taiwanese and US studies of IBD patients reported a notably higher risk of PD relative to controls. ${ }^{78}$ However, neither study considered surveillance bias effects on the measured risk of PD in IBD patients. More recent studies adjusted for the number of healthcare visits to take into account the higher level of surveillance of IBD patients. For example, Swedish IBD patients were 1.3 times more likely to be diagnosed with PD than individuals without IBD, but the higher risk of PD was not significant after adjustment for the number of healthcare visits. ${ }^{9}$ By contrast, Danish and Korean cohort studies revealed an elevated risk of PD in IBD patients that remained significant after adjustment for the number of healthcare visits. ${ }^{1011} 15$

Because age is such an important risk factor for PD, age at IBD diagnosis should be considered when determining the risk for PD. A meta-analysis suggested that patients with onset of IBD over the age of 60 years have a 32\% higher risk of PD than those without IBD, whereas patients diagnosed under the age of 50 did not exhibit this association. ${ }^{16}$ By contrast, another study demonstrated that the higher risk of PD in CD patients was significant only among individuals with age at onset younger than 60 years. ${ }^{11}$ In addition, it is worth noting that older people often take more medication, which might lead to a higher risk of drug-induced parkinsonism; 
the second most common form of parkinsonism in the elderly after idiopathic PD. Medication that interferes with dopamine transmission can cause parkinsonism; accordingly, the most common causative drugs are agents that block dopamine receptors (especially the dopamine D2 receptor), such as antipsychotics, antiemetics, and prokinetic agents. Therefore, the elevated use of antipsychotics among people with IBD is an important consideration to determine whether PD risk is elevated in IBD patients, ${ }^{17}$ as the parkinsonism observed might be secondary to the effect of a drug.

Furthermore, it is often challenging to distinguish idiopathic PD from secondary parkinsonism by administrative data. ${ }^{18}$ ICD codes have been used to identify the presence of PD in population-based studies. However, ICD-8 and ICD-9 do not have separate codes for secondary or atypical parkinsonism, which in ICD-10 is classified as G21 secondary parkinsonism. Therefore, the potential misclassification of drug-induced parkinsonism as idiopathic PD could bias the association between PD and IBD, particularly in elderly IBD patients.

Moreover, the potential role of environmental factors in the association between concomitant IBD and PD has only received limited attention. The effect of smoking would be a very important starting point given its complex interaction(s). Smoking is a risk factor for $C D,{ }^{1}$ but appears to be protective against $U C^{2}$ and PD. ${ }^{3}$ To date, it remains ambiguous whether smoking protects IBD patients from developing PD.

If IBD and PD share disease pathogenesis involving gut inflammation, could medications that treat IBD and control inflammation subsequently decrease the risk of PD? No study to date has examined how IBD activity is related to the risk of PD. Interestingly, IBD patients treated with anti-tumor necrosis factor (TNF) agents had a 78\% reduction in their risk to develop PD, relative to those without anti-TNF treatment. ${ }^{8}$ In addition, a Spanish study using data from the Andalusian Public Health System ${ }^{19}$ reported that PD risk was reduced by $25 \%$ in putative IBD patients who received 5-aminosalicylic acid. Also the use of azathioprine, and corticosteroids is significantly associated with reduced risk of PD. ${ }^{20}$ These observations support the idea that treatment of IBD might decrease subsequent risk of PD. 


\section{Genetic evidence linking IBD and PD}

Consistent with the epidemiological evidence described above, IBD (especially CD) and PD have genetic risk factors in common. ${ }^{12} 2122$ Witoelar et al. analysed genome-wide association studies (GWAS) on patients with PD or any of seven autoimmune disorders (CD, UC, celiac disease, multiple sclerosis, rheumatoid arthritis, psoriasis and type 1 diabetes) to search for pleiotropic gene loci, i.e. markers associated with several disorders. ${ }^{12}$ They detected considerable genetic overlap between PD and autoimmune diseases, with the strongest genome-wide pleiotropic enrichment between PD and CD. Seven PD risk loci were found to overlap with CD (MROH3P, HLA, CCNY, LRRK2, MAPT, SYMPK, and RSPH6A) and four with UC (GUCY1A3, HLA, BTNL2, and TRIM10). ${ }^{12}$

In addition, we evaluated previously identified associations for IBD and PD as listed in the National Human Genome Research Institute GWAS catalog (accessed on 22/06/2020), and looked for overlapping susceptibility loci. ${ }^{23}$ Besides the HLA (human leukocyte antigen) locus, six loci [using $50 \mathrm{~kb}$ on either side of the published single-nucleotide polymorphism (SNP]) are significantly associated with both diseases (Table 3 ). The chromosome $14 q 31.3$ region contains two protein-coding genes: the lysosomal enzyme galactosylceramidase (GALC) and acid-sensing surface receptor G-protein coupled receptor 65 (GPR65); and thus points to lysosome dysfunction as a common mechanism. GALC is a lysosomal enzyme involved in the catabolism of galactosylceramide, and GPR65 plays a role in immune responses by maintaining lysosome function and supporting phagocytosis-mediated intracellular bacteria clearance. ${ }^{2425}$ The most strongly associated lead SNPs tagged in each GWAS analysis, rs8005161 ( $r^{2}=1$ with coding variant rs3742704 located in GPR65) for IBD and rs8005172 for PD (Table 3), were in moderate linkage disequilibrium in an East Asian population $\left(r^{2}=0.47\right.$ in Chinese Dai in Xishuangbanna from Phase 3 of the 1000 Genomes Project) and low linkage disequilibrium in a European population $\left(r^{2}=0.14\right.$ in Utah Residents with Northern and Western European Ancestry from Phase 3 of the 1000 Genomes Project). Although the causal variant responsible for the association of this locus with PD risk remains to be identified, the coding variant GPR65 p.I231L (rs3742704) associated with IBD is related to lysosomal dysfunction, with effects on autophagy and antibacterial defense. ${ }^{26} 27$

The most recent and largest GWAS meta-analysis that studied both PD patients and firstdegree relatives of PD patients as 'proxy-cases' reported 38 novel susceptibility loci including 
FCGR2A and NOD2, which are highly relevant for IBD pathogenesis. ${ }^{28}$ The lead SNP (rs6658353) in the FCGR2A locus in the PD GWAS is in strong linkage disequilibrium $\left(r^{2}>0.9\right.$ in a European population) with a well-characterized nonsynonymous FCGR2A SNP (rs1801274). ${ }^{29}$ The latter is associated with IBD and is shown to affect the recognition of immunoglobulins. ${ }^{30}$ This metaanalysis also identified genome-wide evidence for an association between an intronic NOD2 SNP (rs6500328) and PD susceptibility. NOD2 is the most strongly associated and most consistently replicated CD gene (Table 3). ${ }^{129}$ This new evidence implicates the role of immune responses and microbial sensing in the development of PD.

One of the most interesting genes in the search for a genetic link between IBD and PD is leucine-rich repeat kinase 2 (LRRK2) (Figure 3). LRRK2 is a large (2527 amino acids, 286 kDa) multi-domain protein with a catalytic core, which displays kinase and GTPase activity and is flanked by scaffolding regions. ${ }^{31}$ The $L R R K 2$ gene first attracted attention in 2004, when functional variants in this locus were linked to autosomal dominant PD. ${ }^{32} 33$ Pathogenic missense mutations (e.g. p.G2019S, rs34637584) increase LRRK2 kinase activity. ${ }^{34}$ This has led to the development of LRRK2 kinase inhibitors as potential disease-modifying PD drugs, which are currently being tested in clinical studies. ${ }^{35}$

In addition to PD, LRRK2 is associated with other diseases, including IBD, leprosy, and cancer. ${ }^{31}$ GWAS indeed identified an association between the LRRK2 locus and IBD, and this for several LRRK2 genetic variants (Table 3$)^{29} 36$ including $\mathrm{rs} 4768236^{36}$ which is in perfect linkage disequilibrium with rs3761863 (p.M2397T). LRRK2 p.M2397T has been shown to affect LRRK2 protein turnove. ${ }^{37} 38$ Beyond GWAS, the functional coding variants in LRRK2 (p.G2019S ${ }^{39}$ and p.N2081D ${ }^{22}$ ) have initially been reported to increase the risk of CD in individuals of Ashkenazi Jewish ancestry, probably because these missense mutations are more common in the Ashkenazi population (Figure 3). ${ }^{22} 39$ Hui et al. furthermore reported that LRRK2 p.N2081D (rs33995883) have shared effects on the risks of PD and CD in not only larger cohorts of Jewish but also in individuals of non-Jewish descent. ${ }^{22}$ Moreover, they reported the p.N551K variant (rs7308720, in linkage disequilibrium with p.R1398H (rs7133914) in the GTPase domain) is associated with reduced risk of both CD and PD, while the p.N2081D variant (rs33995883, in the same kinase domain as p.G2019S) is associated with an elevated risk of both CD and PD, although being less strongly associated with PD. Previously reported GWAS associations (Table 3) $)^{29} 36$ in high linkage disequilibrium with p.M2397T (rs3761863) were substantially 
attenuated in a conditional analysis correcting for p.N2081D (rs33995883). ${ }^{22}$ Based on phosphorylation of the LRRK2 substrate RAB10, the protein kinase activity of the p.N2081D risk variant for $C D$ was reported to be elevated, whereas the $\mathrm{p} . \mathrm{R} 1398 \mathrm{H}$ variant, which is protective against CD and PD, leads to increased GTPase activity, resulting in a less active GDPbound LRRK2. ${ }^{22}$ Further insight in the molecular and biological consequences of these shared variants will be important as this might reveal common pathogenic pathways for both diseases. An interesting question that arises is whether the shared effects of $L R R K 2$ mutation(s) in IBD and PD imply also shared therapeutic possibilities of LRRK2 kinase inhibitors. First, the magnitude and direction of effect of the shared LRRK2 variants found through GWAS are largely similar between the two diseases. ${ }^{22}$ However, as will also be discussed in the next paragraph, monogenic causal variants in LRRK2 are only observed in PD patients. Therefore, the therapeutic potential of targeting LRRK2 may be less pronounced in IBD, compared to PD. Second, the distribution and frequency of mutations and genetic susceptibility factors in LRRK2 shows ethnic-specific differences, such as the higher allele frequency of p.G2019S in North African Arabs and the Ashkenazi population, while being monomorphic in Asians..$^{4041}$ Third, the biological consequence(s) of modified LRRK2 activity remain unclear in IBD. Transgenic mice that overexpress $L r r k 2$ are more susceptible to dextran sodium sulfate (DSS)induced colitis. ${ }^{42}$ Interestingly, LRRK2 kinase inhibitors ameliorated DSS-induced colitis, both in control and Lrrk2 transgenic animals, and could block TNF- $\alpha$ production in cultured dendritic cells from CD patients. ${ }^{42}$ Although these findings are very exciting in light of the therapeutic potential of LRRK2 inhibitors for IBD, it will be essential to obtain more insight into the molecular mechanisms shared by the two disorders. Indeed, an earlier study discordantly reported increased susceptibility to IBD in Lrrk2 knockout mice and showed that the CD riskassociated allele encoding LRRK2 p.M2397T results in lower LRRK2 protein levels. ${ }^{38}$ This discrepancy may reflect the complex nature of the biological function of LRRK2 and stresses the need for further investigations with genetic and biological studies to gain insight into the molecular mechanisms of LRRK2 shared by the two disorders.

Although PD and IBD susceptibility has mostly a polygenic origin, a small fraction of patients has monogenic forms of the diseases. In contrast to the genome-wide pleiotropy described above, the genes responsible for monogenic IBD do not overlap with those for monogenic PD. About 50 single genes associated with very early-onset (monogenic) IBD have been identified, 
including ILIOR and XIAP; and most are linked to immune deficiencies. ${ }^{43}$ Mutations in several genes can lead to monogenic PD, including mutations in PINK1, Parkin (both related to mitochondrial function), and ATP13A2 (related to lysosomal function). Some of the monogenic PD genes, such as those encoding for LRRK2 and $\alpha$-synuclein, have also been described as harboring risk variants for sporadic PD. ${ }^{44}$ Mutations in LRRK2 are the most common cause of monogenic $\mathrm{PD}^{3} 45$ (from $<1 \%$ to up to $37 \%$ of familial PD patients, depending on the population ${ }^{45}$ ), but are not linked to monogenic forms of IBD. These genetic variants have large effects on gene function, which have broad implications for the elucidation of disease mechanisms, identification of biomarkers, and targeted therapy. However, shared rare variants causing the monogenic forms of PD and IBD have not yet been reported.

\section{Potential biological mechanisms linking IBD and PD}

\section{Gut-brain axis}

The gut and the brain are connected both physically and biochemically (Figure 4A): the gastrointestinal tract itself contains 500 million neurons, and the vagus nerve, one of the largest nerves connecting the gut and brain, sends signals in both directions. ${ }^{46}$ The gut and brain are also connected through neurotransmitters, including serotonin and gammaaminobutyric acid. ${ }^{46}$ In addition, many studies have focused on the effects of the gut microbiota on the brain, as a node of this axis. ${ }^{47} \mathrm{~A}$ pivotal study showed that germ-free mice exhibit an exaggerated response to stress induced by physical restraint, implying that the microbiota influence the hypothalamic-pituitary-adrenal axis, the central stress-response system. ${ }^{48}$ This is consistent with findings in mice showing that a lack of conventional microbiota affects behaviour and gene expression in the brain. ${ }^{49-51}$ Vice versa, stress can increase intestinal permeability in murine models, ${ }^{52}$ which might facilitate intestinal translocation of bacteria. Together, these animal studies clearly show that the gut microenvironment can affect behaviour through various compounds produced by the gut and its microbes (gut-to-brain), whereas stress can perturb the composition of the microbiota (brain-to-gut).

The interaction between brain and gut is an important topic in the IBD field. ${ }^{6}$ Psychological stress in IBD patients can influence inflammatory activity by increasing the levels of serum and mucosal inflammatory cytokines (Figure 4A)..$^{53}$ On the other hand, the use of anti-TNF agents is associated with improvement in depression scores. ${ }^{54}$ Recently, Gracie et al. prospectively 
evaluated the bidirectional link between the brain and gut in 405 IBD patients over a minimum of 2 years. ${ }^{55}$ In inactive IBD, baseline psychological comorbidity was associated with a 2-fold increase in the risk of disease relapse and up-titration of medical therapy. ${ }^{55}$ Despite the limited number of well-designed studies with IBD patients, these data suggest that anxiety or depression might worsen the natural history of IBD, leading to a substantial decrease in quality of life. ${ }^{6}$

In addition, accumulating evidence suggests that chronic gastrointestinal inflammation, a characteristic of uncontrolled IBD, can lead to neuroinflammation via the gut-brain axis (Figure 4B). ${ }^{56} 57$ This is also in line with elevated stool calprotectin (gut inflammatory marker) levels reported in PD patients. ${ }^{58}$ Notably in this regard, independent studies have associated DSS-induced colitis in rodents with dopaminergic cell death. ${ }^{59}$ Chronic intestinal inflammation might trigger aggregation of $\alpha$-synuclein in the gut, which then spreads to the brain using the vagal nerve. ${ }^{60}$ Interest in this field was paved by Braak's hypothesis, ${ }^{61}$ which posits that pathogens enter the gut through the nasal cavity or mouth; and can initiate Lewy pathology in the nose and gut, preceding changes in the brain.

This hypothesis is supported by several studies. First, Lewy bodies can be observed in the gut of PD patients up to 20 years before PD diagnosis. ${ }^{60}$ Therefore, it is reasonable to speculate that $\alpha$-synuclein starts to aggregate in the gut in the early stages, even before the first PD motor symptoms appear. Second, in animal studies, the injection of exogenous $\alpha$-synuclein fibrils into the gut causes conversion of endogenous $\alpha$-synuclein into a pathologic species that spreads to the brain. ${ }^{62} 63$ Third, experimental ${ }^{64}$ and clinical ${ }^{65} 66$ data reveal that vagotomy can decrease the risk of PD. Finally, appendectomy is associated with a lower risk of PD, most likely related to intraneuronal $\alpha$-synuclein aggregates in the appendix. ${ }^{66}$ However, this observation has not been replicated by independent studies. ${ }^{66}$ Taken together, these observations suggest that Lewy pathology can start in the gastrointestinal tract after which it is spread via the vagus nerve to the brain, where it contributes to the pathogenesis and progression of PD (Figure 4B). Expression of $\alpha$-synuclein in both the gut and brain of PD patients represents a possible link between PD and IBD. However, the precise mechanism and role of enteric $\alpha$-synuclein remain unknown. ${ }^{4}$ Also, it is not clear whether $\alpha$-synuclein aggregation is a cause or a consequence of gut inflammation. In general, $\alpha$-synuclein is a ubiquitously expressed protein, and its presence in the gut is also common in healthy individuals. Despite the observation that $\alpha$ - 
synuclein protein levels increase with age, it is yet unclear whether this should be considered as a pathological condition. ${ }^{67}$ Recent studies suggest that $\alpha$-synuclein plays a role in the immune response in the gut via the enteric nervous system, which is essential for microbeinduced immune responses in the gut. ${ }^{568}$ Conversely, PD-related neurodegeneration may also play a role in gut inflammation. Injection of the neurotoxin 6-hydroxydopamine (6-OHDA) into the substantia nigra can produce lesions in nigral dopaminergic neurons, and is therefore often used to model PD. This central neuronal degeneration was shown to be followed by bowel inflammation associated with increased oxidative stress, pro-inflammatory cytokine levels, and activation of enteric glia and inflammatory cells. ${ }^{69}$ This might be related to the observation that 6-OHDA can also affect cholinergic neurons of the dorsal motor nucleus of the vagus. ${ }^{59}$

More insight in how gut inflammation contributes to central neuronal pathology, and vice versa, will be pivotal to better understand disease pathogenesis and thus to provide new potential therapeutic targets. In this context, identifying the risk factors associated with developing PD among IBD patients will be clinically important. To gain further insight into the risk factors among patients with IBD, we may start to explore which group of patients with PD have a higher gut inflammatory burden. It has been reported that the level of stool calprotectin in PD patients is elevated compared to the general population. ${ }^{58}$ Therefore, stool calprotectin levels might be informative to select a priority PD patient group that is more vulnerable to intestinal inflammation to investigate. For example, it could be further investigated if this selected group of PD patients shows more genetic overlap with IBD patients. Also, intestinal tissue of PD patients with high fecal calprotectin level would be very valuable to further unravel the molecular mechanisms underlying both pathologies. Below, we further discuss molecular mechanisms that may underlie the link between gut inflammation in IBD and neurodegeneration in PD, and how this relates to (dys)fynction of LRRK2, given the importance of this protein in the IBD-PD connection. ${ }^{56}$

\section{Possible biological connections}

\subsection{Gut microbiota}

Gut microbiota may play a role in the communication between the gut and the brain. It is commonly accepted that IBD results from a defective mucosal immune response to gut microbiota in genetically susceptible individuals. ${ }^{12}$ Multiple lines of evidence have recently 
also emerged on the role of gut microbiota in the pathogenesis of PD (extensively reviewed in $\left.{ }^{57}\right)$. Indeed, microbial dysbiosis can lead to increased gut mucosal permeability, inflammation, impaired short-chain fatty acid balance, and/or oxidative stress, which in turn may trigger $\alpha$ synuclein aggregation (Figure 4B). ${ }^{70}$ In an $\alpha$-synuclein transgenic PD mouse model, transplanted fecal microbiota from PD patients exacerbated motor dysfunction to a significantly greater degree than microbiota from healthy individuals. ${ }^{71}$ In addition, gut infection may also play an important role in the development of PD symptoms. Although lossof-function mutations in PINK1 can cause PD in patients, Pink1-knockout mice do not develop PD-like symptoms and were reported to be generally healthy. This might be related to the fact that these mice were raised under germ-free conditions and thus lack gut microbes. Indeed, infection of young Pink1-knockout mice with gram-negative bacteria that cause mild intestinal symptoms was sufficient to trigger PD-like symptoms later in life. ${ }^{72}$ This is in line with epidemiological data showing that gastrointestinal infections are associated with an elevated risk of PD. ${ }^{6873}$ Future studies should seek to determine whether altering intestinal microbiota (e.g. via probiotics/prebiotics or fecal transplantation) can exert beneficial effects on PD pathology given that modulation of gut microbiota represents a potential novel strategy for treating PD. ${ }^{57}$

\section{2. (Auto)immunity}

The role of the immune system in the development of IBD is well established. But also in PD, although the cause of dopaminergic neuron degeneration remains largely unknown, emerging evidence suggests that neuroinflammation as well as the peripheral immune system contribute to PD pathogenesis (extensively reviewed in ${ }^{74}$ ). Indeed, the brain is no longer considered an immune-privileged organ, and evidence from animal models and patients strongly suggests the involvement of the innate and adaptive immune system. ${ }^{74} 75$ The involvement of the adaptive immune system is also implied by the strong association between SNPs at the HLA locus, which spans at least ten immune-related genes, and the risk of developing PD. ${ }^{28} 76$ Infiltrated T-cells have been detected in the substantia nigra of PD patients, and specific T-cells from PD patients recognize $\alpha$-synuclein-derived fragments as antigenic epitopes, ${ }^{77}$ which suggests T-cell activation by $\alpha$-synuclein and autoimmunity.

Interestingly, $\alpha$-synuclein can be internalized by lipopolysaccharides (LPS, which are components of the outer membrane of most gram-negative bacteria)-primed inflammatory 
monocytes, favoring dissemination from the periphery toward the brain. ${ }^{78}$ In addition, PINK1 and Parkin can suppress the presentation of mitochondrial antigens induced by exposure to LPS. $^{72} 79$ In Pink1\% mice, gram-negative bacteria gut infection triggers an immune response that results in the production of $\mathrm{CD}^{+} \mathrm{T}$-cells specific for mitochondrial antigens, which is repressed in wild type mice. In infected Pink1\% mice, these autoreactive T-cells are also present in the brain. Thus, the detrimental effect of PINK1 on PD may be triggered in the gut and subsequently spread to the brain, which implies that a part of PD pathogenesis may be related to autoimmunity that is initiated in the gastrointestinal tract.

Emerging evidence points to an important role for LRRK2 in peripheral immune cells. In humans, LRRK2 is expressed at the highest levels in cells of the myeloid lineage such as monocytes and dendritic cells. Whether and how LRRK2 can contribute to PD pathology through its role in peripheral immune responses has yet to be established and was recently discussed by Cabezudo et al. ${ }^{80}$ Interestingly, there might be a role for LRRK2 in intestinal inflammation. LRRK2 expression is elevated in intestinal biopsy specimens and dendritic cells from CD patients. ${ }^{42} 81$ Further evidence for a regulatory role for LRRK2 in gut inflammation comes from studies using Lrrk2 knockout mice, which present with increased neuropeptide levels accompanied by an increment of $\operatorname{lgA}$ levels, ${ }^{82}$ as well as increased susceptibility to DSSinduced colitis via enhanced NFAT (nuclear factor of activated T cells) signaling in myeloid cells. ${ }^{38}$ Intriguingly, also overexpression of wild-type LRRK2 in mice was shown to increase the severity of colitis, which was associated with increased NF-KB activation in lamina propria dendritic cells and proinflammatory cytokine secretion. ${ }^{42} \mathrm{~A}$ study using transgenic LRRK2 p.G2019S rats showed that also pathogenic PD-causing LRRK2 variants can affect inflammatory processes in the gut. ${ }^{83}$ Under inflammatory conditions, these rats exhibited reduced bone marrow myelopoiesis and a dysregulated peripheral myeloid phenotype, leading to suppressed Th17-cell activity with abnormally high levels of phylum Bacteroidetes in the intestine. ${ }^{83}$ Such disturbed peripheral immune homeostasis might affect inflammatory responses in multiple organs, including intestine and brain. Taken together, these data strongly suggest a role for LRRK2 in peripheral immune cells, although it has yet to be established to what extent dysregulated periphery immune responses contribute to the LRRK2-related risk of developing IBD and PD. 


\subsection{Mitochondria}

As vital organelles involved in energy production, mitochondria play key roles in redox homeostasis, and they undergo fission and fusion in response to changes in metabolism and oxidative stress. Mitochondrial dysfunction plays a pivotal role in sporadic and familial PD, and is believed to contribute to neuronal death. ${ }^{84}$ In particular, the elimination of damaged mitochondria, collectively called mitophagy, is directly connected to PD pathogenesis given the central role of PINK1 and Parkin in this cellular process. ${ }^{85}$ Also LRRK2 is reported to be involved in mitophagy, although its exact role in this context is still under debate given that both increased and delayed mitophagy have been attributed to the G2019S variant. ${ }^{86} 87$

Because maintenance of the epithelial barrier is energy-dependent, mitochondrial dysfunction is also predicted to contribute to the development and progress of IBD, though the underlying mechanisms remain unclear. Indeed, hallmarks of mitochondrial dysfunction, such as oxidative stress and impaired adenosine triphosphate (ATP) production, are observed in the gut of IBD patients. ${ }^{88}$ This is consistent with several reports of altered mitochondrial function in the intestinal epithelium of mouse models of colitis as well as IBD patients. ${ }^{88} 89$ Recent studies have linked mitochondrial dysfunction to Paneth cells, which are specialized epithelial cells in the small intestine that secrete enteric antimicrobial peptides. Dysfunctional cells can display impaired secretion that can ultimately result in ileitis. ${ }^{90} 91$

Mitochondrial dysfunction and dysbiosis of the gut microbiota are closely related. Microbiota signaling to the mitochondria can alter mitochondrial metabolism, activate immune cells, induce inflammasome signaling, and alter epithelial barrier function. ${ }^{89}$ Together, these effects may induce intestinal inflammation, contributing to IBD and PD pathogenesis. Thus, a better understanding of how abnormalities in mitochondrial dynamics and metabolism within the intestinal epithelium contribute to disease will improve our overall understanding of the link between PD and IBD, and may provide mitochondria-based treatment strategies for both diseases.

\subsection{Autophagy and lysosomal function}

The observation that Lewy bodies, one of the hallmarks of PD, consist of misfolded protein, with $\alpha$-synuclein aggregates as main constituent, strongly implicates autophagic and/or lysosomal dysfunction in PD pathogenesis. Dysfunction in these pathways is also heavily involved in IBD, leading to compromised intestinal epithelial barrier function, a disrupted 
microbiome, and defective antibacterial peptide secretion. ${ }^{25}$ Consistent with this, numerous susceptibility loci in both $\mathrm{PD}^{28}$ and $\mathrm{IBD}^{29}$ have been functionally implicated in autophagy and lysosome function.

Substantial evidence points to a role for LRRK2 in the autophagic/lysosomal pathway. LRRK2 co-localizes with the autophagic protein microtubule-associated protein 1A/1B-light chain 3 (LC3), phosphorylates the adaptor protein p62 and has been suggested to play a crucial role in maintaining autophagic equilibrium. ${ }^{86} 92$ When it comes to the involvement of LRRK2 in the gut, LRRK2 expression is found to be high in Paneth cells, which provide host defense against microbes in the small intestine. LRRK2 deficiency causes specific deprivation of lysozyme in Paneth cells which rendered LRRK2 knockout mice more susceptible to intestinal infection with Listeria monocytogenes, with increased bacterial loads in fecal samples and liver, compared to wild-type mice. ${ }^{93}$ The observation that LRRK2 acts downstream of NOD2 in regulating lysozyme sorting is very relevant in the link between IBD and PD, given that the NOD2 locus is strongly associated with IBD, and more recently also with PD risk (Table 3).28 29 Moreover, LRRK2 is shown to be a positive regulator of inflammatory cytokine induction in macrophages through the Nod1/2-Rip2 pathway, which depend on several cellular processes including autophagy. ${ }^{25}$ Through its role in lysosomal function, LRRK2 might contribute to control intestinal infections, which in turn might be closely related to its effect on microbial composition in the gut. To what extent this function is related to neurodegeneration, can be affected by pathogenic PD mutations, or can be altered by LRRK2 kinase inhibition has yet to be resolved.

\section{Conclusion}

Although PD and IBD seem very different disorders at first glance, they have important similarities (Table 1). Both are common and complex chronic diseases with heterogeneous clinical features and a progressive nature. Because both PD and IBD remain incurable, they represent significant public health challenges worldwide. A growing body of evidence points to a link between PD and IBD, highlighting the relevance of chronic inflammation and the gutbrain axis in the pathogenesis of these two diseases (Figure 2 and Figure 4). Several large population studies have shown that IBD patients are at substantially higher risk of PD. Given that PD most often affects the elderly, the number of individuals living with PD rises as populations age. At the same time, the negligible impact of IBD on mortality leads to 
compounding prevalence of IBD, resulting in a rapid rise of IBD in the elderly population. This, in turn, can result in a higher burden of patients suffering from concomitant IBD and PD.

Given the role of gut inflammation in the pathogenesis of PD, it will be interesting to determine whether controlling IBD activity can reduce the risk of subsequent development of PD (Figure 4B). Moreover, a subset of PD patients might be genetically predisposed to gut inflammation and microbial dysbiosis. In this regard, the identification of risk factors associated with prodromal phases of PD among IBD patients may allow for early interventions that could modify or slow down PD progression. On the other hand, knowledge of shared mechanisms (e.g. TNF) and shared genetic variants (e.g. LRRK2) will shed light on the complexities of these diseases and facilitate the development of therapeutic strategies. More practically, pre-existing medications for treatment of IBD could be cautiously repurposed into PD therapeutics, or vice versa.

Emerging evidence supports the idea of an important link between these two diseases (Box 1). Still, it should be noted that further research will be indispensable to provide definite evidence for a robust and bona fide association between IBD and PD. A comprehensive understanding of the relationship between both disorders will require the expertise of researchers from a wide range of disciplines (neurology, gastroenterology, epidemiology, molecular biology, genetics, and others); however, given the links between IBD and PD and the increasing burden associated with both diseases, it is important to answer these questions in the near future. Collective efforts will accelerate the discovery of novel disease-associated pathways and ultimately facilitate the identification of potential therapeutic targets. 


\section{References}

1. Baumgart DC, Sandborn WJ. Crohn's disease. Lancet 2012;380:1590-605.

2. Ordas I, Eckmann L, Talamini M, et al. Ulcerative colitis. Lancet 2012;380:1606-19.

3. Kalia LV, Lang AE. Parkinson's disease. Lancet 2015;386:896-912.

4. Wong YC, Krainc D. alpha-synuclein toxicity in neurodegeneration: mechanism and therapeutic strategies. Nat Med 2017;23:1-13.

5. Yoo BB, Mazmanian SK. The Enteric Network: Interactions between the Immune and Nervous Systems of the Gut. Immunity 2017;46:910-26.

6. Gracie DJ, Hamlin PJ, Ford AC. The influence of the brain-gut axis in inflammatory bowel disease and possible implications for treatment. Lancet Gastroenterol Hepatol 2019;4:632-42.

7. Lin JC, Lin CS, Hsu CW, et al. Association Between Parkinson's Disease and Inflammatory Bowel Disease: a Nationwide Taiwanese Retrospective Cohort Study. Inflamm Bowel Dis 2016;22:1049-55.

8. Peter I, Dubinsky M, Bressman S, et al. Anti-Tumor Necrosis Factor Therapy and Incidence of Parkinson Disease Among Patients With Inflammatory Bowel Disease. JAMA Neurol 2018;75:939-46.

9. Weimers P, Halfvarson J, Sachs MC, et al. Inflammatory Bowel Disease and Parkinson's Disease: A Nationwide Swedish Cohort Study. Inflamm Bowel Dis 2019;25:111-23.

10. Villumsen $M, A z n a r ~ S, ~ P a k k e n b e r g ~ B$, et al. Inflammatory bowel disease increases the risk of Parkinson's disease: a Danish nationwide cohort study 1977-2014. Gut 2019;68:1824.

11. Park S, Kim J, Chun J, et al. Patients with Inflammatory Bowel Disease Are at an Increased Risk of Parkinson's Disease: A South Korean Nationwide Population-Based Study. J Clin Med 2019;8

12. Witoelar A, Jansen IE, Wang Y, et al. Genome-wide Pleiotropy Between Parkinson Disease and Autoimmune Diseases. JAMA Neurol 2017;74:780-92.

13. Camacho-Soto A, Gross A, Searles Nielsen S, et al. Inflammatory bowel disease and risk of Parkinson's disease in Medicare beneficiaries. Parkinsonism Relat Disord 2018;50:2328.

14. Fasano A, Visanji NP, Liu LW, et al. Gastrointestinal dysfunction in Parkinson's disease. Lancet Neurol 2015;14:625-39.

15. Villumsen M, Aznar S, Pakkenberg B, et al. Authors' response: Association between IBD and Parkinson's disease: seek and you shall find? Gut 2019;68:1722.

16. Wan QY, Zhao R, Wu XT. Older patients with IBD might have higher risk of Parkinson's disease. Gut 2020;69:193-94.

17. Bernstein $\mathrm{CN}$, Hitchon CA, Walld R, et al. Increased Burden of Psychiatric Disorders in Inflammatory Bowel Disease. Inflamm Bowel Dis 2019;25:360-68.

18. Wermuth L, Cui X, Greene N, et al. Medical Record Review to Differentiate between Idiopathic Parkinson's Disease and Parkinsonism: A Danish Record Linkage Study with 10 Years of Follow-Up. Parkinsons Dis 2015;2015:781479.

19. Pinel Rios J, Madrid Navarro CJ, Perez Navarro MJ, et al. Association of Parkinson's disease and treatment with aminosalicylates in inflammatory bowel disease: a cross-sectional study in a Spain drug dispensation records. BMJ Open 2019;9:e025574.

20. Racette BA, Gross A, Vouri SM, et al. Immunosuppressants and risk of Parkinson disease. Ann Clin Transl Neurol 2018;5:870-75. 
21. Nalls MA, Saad M, Noyce AJ, et al. Genetic comorbidities in Parkinson's disease. Hum Mol Genet 2014;23:831-41.

22. Hui KY, Fernandez-Hernandez $\mathrm{H}$, Hu J, et al. Functional variants in the LRRK2 gene confer shared effects on risk for Crohn's disease and Parkinson's disease. Sci Transl Med 2018;10

23. Buniello A, MacArthur JAL, Cerezo M, et al. The NHGRI-EBI GWAS Catalog of published genome-wide association studies, targeted arrays and summary statistics 2019. Nucleic Acids Res 2019;47:D1005-D12.

24. Ysselstein D, Shulman JM, Krainc D. Emerging links between pediatric lysosomal storage diseases and adult parkinsonism. Mov Disord 2019;34:614-24.

25. Larabi A, Barnich N, Nguyen HTT. New insights into the interplay between autophagy, gut microbiota and inflammatory responses in IBD. Autophagy 2020;16:38-51.

26. Lassen KG, McKenzie $\mathrm{Cl}$, Mari M, et al. Genetic Coding Variant in GPR65 Alters Lysosomal pH and Links Lysosomal Dysfunction with Colitis Risk. Immunity 2016;44:1392-405.

27. Tcymbarevich IV, Eloranta JJ, Rossel JB, et al. The impact of the rs 8005161 polymorphism on $\mathrm{G}$ protein-coupled receptor GPR65 (TDAG8) pH-associated activation in intestinal inflammation. BMC Gastroenterol 2019;19:2.

28. Nalls $\mathrm{MA}$, Blauwendraat $\mathrm{C}$, Vallerga $\mathrm{CL}$, et al. Identification of novel risk loci, causal insights, and heritable risk for Parkinson's disease: a meta-analysis of genome-wide association studies. Lancet Neurol 2019;18:1091-102.

29. de Lange KM, Moutsianas L, Lee JC, et al. Genome-wide association study implicates immune activation of multiple integrin genes in inflammatory bowel disease. Nat Genet 2017;49:256-61.

30. Lu J, Marnell LL, Marjon KD, et al. Structural recognition and functional activation of FcgammaR by innate pentraxins. Nature 2008;456:989-92.

31. Lewis PA. Leucine rich repeat kinase 2: a paradigm for pleiotropy. J Physiol 2019;597:351121.

32. Paisan-Ruiz C, Jain S, Evans EW, et al. Cloning of the gene containing mutations that cause PARK8-linked Parkinson's disease. Neuron 2004;44:595-600.

33. Zimprich A, Biskup $S$, Leitner $P$, et al. Mutations in LRRK2 cause autosomal-dominant parkinsonism with pleomorphic pathology. Neuron 2004;44:601-7.

34. Islam MS, Moore DJ. Mechanisms of LRRK2-dependent neurodegeneration: role of enzymatic activity and protein aggregation. Biochem Soc Trans 2017;45:163-72.

35. Tolosa E, Vila M, Klein C, et al. LRRK2 in Parkinson disease: challenges of clinical trials. Nature reviews Neurology 2020;16:97-107.

36. Liu JZ, van Sommeren S, Huang H, et al. Association analyses identify 38 susceptibility loci for inflammatory bowel disease and highlight shared genetic risk across populations. Nat Genet 2015;47:979-86.

37. Fava VM, Manry J, Cobat A, et al. A Missense LRRK2 Variant Is a Risk Factor for Excessive Inflammatory Responses in Leprosy. PLoS Negl Trop Dis 2016;10:e0004412.

38. Liu Z, Lee J, Krummey S, et al. The kinase LRRK2 is a regulator of the transcription factor NFAT that modulates the severity of inflammatory bowel disease. Nat Immunol 2011;12:1063-70.

39. Rivas MA, Avila BE, Koskela J, et al. Insights into the genetic epidemiology of Crohn's and rare diseases in the Ashkenazi Jewish population. PLoS Genet 2018;14:e1007329.

40. Ozelius LJ, Senthil G, Saunders-Pullman R, et al. LRRK2 G2019S as a cause of Parkinson's disease in Ashkenazi Jews. N Engl J Med 2006;354:424-5. 
41. Lesage S, Dürr A, Tazir M, et al. LRRK2 G2019S as a cause of Parkinson's disease in North African Arabs. N Engl J Med 2006;354:422-3.

42. Takagawa T, Kitani A, Fuss I, et al. An increase in LRRK2 suppresses autophagy and enhances Dectin-1-induced immunity in a mouse model of colitis. Sci Transl Med 2018;10

43. Uhlig HH, Schwerd T, Koletzko S, et al. The diagnostic approach to monogenic very early onset inflammatory bowel disease. Gastroenterology 2014;147:990-1007 e3.

44. Bandres-Ciga S, Diez-Fairen M, Kim JJ, et al. Genetics of Parkinson's disease: An introspection of its journey towards precision medicine. Neurobiol Dis 2020;137:104782.

45. Puschmann A. Monogenic Parkinson's disease and parkinsonism: clinical phenotypes and frequencies of known mutations. Parkinsonism Relat Disord 2013;19:407-15.

46. Mayer EA. Gut feelings: the emerging biology of gut-brain communication. Nat Rev Neurosci 2011;12:453-66.

47. Rooks MG, Garrett WS. Gut microbiota, metabolites and host immunity. Nat Rev Immunol 2016;16:341-52.

48. Sudo N, Chida Y, Aiba Y, et al. Postnatal microbial colonization programs the hypothalamicpituitary-adrenal system for stress response in mice. J Physiol 2004;558:263-75.

49. Hsiao EY, McBride SW, Hsien S, et al. Microbiota modulate behavioral and physiological abnormalities associated with neurodevelopmental disorders. Cell 2013;155:1451-63.

50. Valles-Colomer M, Falony G, Darzi Y, et al. The neuroactive potential of the human gut microbiota in quality of life and depression. Nat Microbiol 2019;4:623-32.

51. Schretter CE, Vielmetter J, Bartos I, et al. A gut microbial factor modulates locomotor behaviour in Drosophila. Nature 2018;563:402-06.

52. Santos J, Saunders PR, Hanssen NP, et al. Corticotropin-releasing hormone mimics stressinduced colonic epithelial pathophysiology in the rat. Am J Physiol 1999;277:G391-9.

53. Mawdsley JE, Macey MG, Feakins RM, et al. The effect of acute psychologic stress on systemic and rectal mucosal measures of inflammation in ulcerative colitis. Gastroenterology 2006;131:410-9.

54. Horst $S$, Chao A, Rosen $M$, et al. Treatment with immunosuppressive therapy may improve depressive symptoms in patients with inflammatory bowel disease. Dig Dis Sci 2015;60:465-70.

55. Gracie DJ, Guthrie EA, Hamlin PJ, et al. Bi-directionality of Brain-Gut Interactions in Patients With Inflammatory Bowel Disease. Gastroenterology 2018;154:1635-46 e3.

56. Becker A, Fassbender K, Oertel WH, et al. A punch in the gut - Intestinal inflammation links environmental factors to neurodegeneration in Parkinson's disease. Parkinsonism Relat Disord 2019;60:43-45.

57. Dutta SK, Verma S, Jain V, et al. Parkinson's Disease: The Emerging Role of Gut Dysbiosis, Antibiotics, Probiotics, and Fecal Microbiota Transplantation. J Neurogastroenterol Motil 2019;25:363-76.

58. Schwiertz A, Spiegel J, Dillmann U, et al. Fecal markers of intestinal inflammation and intestinal permeability are elevated in Parkinson's disease. Parkinsonism Relat Disord 2018;50:104-07.

59. Rolli-Derkinderen M, Leclair-Visonneau L, Bourreille $A$, et al. Is Parkinson's disease a chronic low-grade inflammatory bowel disease? J Neurol 2019 
60. Stokholm MG, Danielsen EH, Hamilton-Dutoit SJ, et al. Pathological alpha-synuclein in gastrointestinal tissues from prodromal Parkinson disease patients. Ann Neurol 2016;79:940-9.

61. Braak H, Ghebremedhin E, Rub U, et al. Stages in the development of Parkinson's diseaserelated pathology. Cell Tissue Res 2004;318:121-34.

62. Holmqvist S, Chutna O, Bousset L, et al. Direct evidence of Parkinson pathology spread from the gastrointestinal tract to the brain in rats. Acta Neuropathol 2014;128:805-20.

63. Kim S, Kwon SH, Kam TI, et al. Transneuronal Propagation of Pathologic alpha-Synuclein from the Gut to the Brain Models Parkinson's Disease. Neuron 2019;103:627-41 e7.

64. Shprecher DR, Derkinderen P. Parkinson disease: the enteric nervous system spills its guts. Neurology 2012;78:683; author reply 83.

65. Liu B, Fang F, Pedersen NL, et al. Vagotomy and Parkinson disease: A Swedish registerbased matched-cohort study. Neurology 2017;88:1996-2002.

66. Girard-Madoux MJH, Gomez de Aguero M, Ganal-Vonarburg SC, et al. The immunological functions of the Appendix: An example of redundancy? Semin Immunol 2018;36:31-44.

67. Antunes L, Frasquilho S, Ostaszewski M, et al. Similar alpha-Synuclein staining in the colon mucosa in patients with Parkinson's disease and controls. Mov Disord 2016;31:156770.

68. Stolzenberg E, Berry D, Yang, et al. A Role for Neuronal Alpha-Synuclein in Gastrointestinal Immunity. J Innate Immun 2017;9:456-63.

69. Pellegrini $C$, Fornai $M$, Colucci $R$, et al. Alteration of colonic excitatory tachykininergic motility and enteric inflammation following dopaminergic nigrostriatal neurodegeneration. J Neuroinflammation 2016;13:146.

70. Dalile B, Van Oudenhove L, Vervliet B, et al. The role of short-chain fatty acids in microbiota-gut-brain communication. Nature reviews Gastroenterology \& hepatology 2019;16:461-78.

71. Sampson TR, Debelius JW, Thron T, et al. Gut Microbiota Regulate Motor Deficits and Neuroinflammation in a Model of Parkinson's Disease. Cell 2016;167:1469-80 e12.

72. Matheoud D, Cannon T, Voisin A, et al. Intestinal infection triggers Parkinson's disease-like symptoms in Pink1(-/-) mice. Nature 2019;571:565-69.

73. Nerius M, Doblhammer G, Tamguney G. GI infections are associated with an increased risk of Parkinson's disease. Gut 2020;69:1154-56.

74. Tan EK, Chao YX, West A, et al. Parkinson disease and the immune system - associations, mechanisms and therapeutics. Nature reviews Neurology 2020;16:303-18.

75. Fuzzati-Armentero MT, Cerri S, Blandini F. Peripheral-Central Neuroimmune Crosstalk in Parkinson's Disease: What Do Patients and Animal Models Tell Us? Front Neurol 2019;10:232.

76. Hamza TH, Zabetian CP, Tenesa A, et al. Common genetic variation in the HLA region is associated with late-onset sporadic Parkinson's disease. Nat Genet 2010;42:781-5.

77. Sulzer D, Alcalay RN, Garretti F, et al. T cells from patients with Parkinson's disease recognize alpha-synuclein peptides. Nature 2017;546:656-61.

78. Peralta Ramos JM, Iribarren P, Bousset L, et al. Peripheral Inflammation Regulates CNS Immune Surveillance Through the Recruitment of Inflammatory Monocytes Upon Systemic alpha-Synuclein Administration. Front Immunol 2019;10:80.

79. Matheoud D, Sugiura A, Bellemare-Pelletier A, et al. Parkinson's Disease-Related Proteins PINK1 and Parkin Repress Mitochondrial Antigen Presentation. Cell 2016;166:314-27. 
80. Cabezudo D, Baekelandt V, Lobbestael E. Multiple-Hit Hypothesis in Parkinson's Disease: LRRK2 and Inflammation. Front Neurosci 2020;14:376.

81. Gardet A, Benita Y, Li C, et al. LRRK2 is involved in the IFN-gamma response and host response to pathogens. J Immunol 2010;185:5577-85.

82. Maekawa $T$, Shimayama $H$, Tsushima $H$, et al. LRRK2: An Emerging New Molecule in the Enteric Neuronal System That Quantitatively Regulates Neuronal Peptides and IgA in the Gut. Dig Dis Sci 2017;62:903-12.

83. Park J, Lee JW, Cooper SC, et al. Parkinson disease-associated LRRK2 G2019S transgene disrupts marrow myelopoiesis and peripheral Th17 response. J Leukoc Biol 2017;102:1093-102.

84. Abeliovich A, Gitler AD. Defects in trafficking bridge Parkinson's disease pathology and genetics. Nature 2016;539:207-16.

85. Sliter DA, Martinez J, Hao L, et al. Parkin and PINK1 mitigate STING-induced inflammation. Nature 2018;561:258-62.

86. Roosen DA, Cookson MR. LRRK2 at the interface of autophagosomes, endosomes and lysosomes. Mol Neurodegener 2016;11:73.

87. Hsieh CH, Shaltouki A, Gonzalez AE, et al. Functional Impairment in Miro Degradation and Mitophagy Is a Shared Feature in Familial and Sporadic Parkinson's Disease. Cell Stem Cell 2016;19:709-24.

88. Rath E, Moschetta A, Haller D. Mitochondrial function - gatekeeper of intestinal epithelial cell homeostasis. Nature reviews Gastroenterology \& hepatology 2018;15:497-516.

89. Mottawea W, Chiang CK, Muhlbauer M, et al. Altered intestinal microbiota-host mitochondria crosstalk in new onset Crohn's disease. Nat Commun 2016;7:13419.

90. Khaloian S, Rath E, Hammoudi N, et al. Mitochondrial impairment drives intestinal stem cell transition into dysfunctional Paneth cells predicting Crohn's disease recurrence. Gut 2020:gutjnl-2019-319514.

91. Jackson DN, Panopoulos M, Neumann WL, et al. Mitochondrial dysfunction during loss of prohibitin 1 triggers Paneth cell defects and ileitis. Gut 2020:gutjnl-2019-319523.

92. Tong $\mathrm{Y}$, Yamaguchi $\mathrm{H}$, Giaime $\mathrm{E}$, et al. Loss of leucine-rich repeat kinase 2 causes impairment of protein degradation pathways, accumulation of alpha-synuclein, and apoptotic cell death in aged mice. Proc Natl Acad Sci U S A 2010;107:9879-84.

93. Zhang $\mathrm{Q}$, Pan $\mathrm{Y}$, Yan $\mathrm{R}$, et al. Commensal bacteria direct selective cargo sorting to promote symbiosis. Nat Immunol 2015;16:918-26.

94. Ng SC, Shi HY, Hamidi N, et al. Worldwide incidence and prevalence of inflammatory bowel disease in the 21st century: a systematic review of population-based studies. Lancet 2018;390:2769-78.

95. Xu F, Dahlhamer JM, Zammitti EP, et al. Health-Risk Behaviors and Chronic Conditions Among Adults with Inflammatory Bowel Disease - United States, 2015 and 2016. MMWR Morb Mortal Wkly Rep 2018;67:190-95.

96. Pringsheim T, Jette N, Frolkis A, et al. The prevalence of Parkinson's disease: a systematic review and meta-analysis. Mov Disord 2014;29:1583-90. 
Table 1. Differences and commonalities between inflammatory bowel disease and Parkinson's disease.

\begin{tabular}{|c|c|c|}
\hline & Inflammatory bowel disease & Parkinson's disease \\
\hline Organ mainly affected & Gut & Brain \\
\hline Prevalence & Up to $1.3 \%{ }^{9495}$ & $0.3 \%{ }^{96}$ \\
\hline Peak incidence & $15-35$ years & Rising with age \\
\hline Risk factors & Family history, Smoking (CD) & $\begin{array}{l}\text { Ageing, Family history, Exposure } \\
\text { to toxins }\end{array}$ \\
\hline Typical symptoms & $\begin{array}{l}\text { Diarrhea, Abdominal pain and } \\
\text { cramping, Bloody stool, } \\
\text { Unintended weight loss }\end{array}$ & $\begin{array}{l}\text { Tremor, Impaired posture and } \\
\text { balance, Bradykinesia, Muscle } \\
\text { stiffness, Loss of spontaneous } \\
\text { movements }\end{array}$ \\
\hline Histologic features & $\begin{array}{l}\text { Non caseating granulomas for CD } \\
\text { and cryptitis/crypt abscesses for } \\
\text { UC }\end{array}$ & $\begin{array}{l}\text { Neurodegeneration in the } \\
\text { substantia nigra pars compacta, } \\
\text { Alpha-synuclein positive neuronal } \\
\text { inclusions named Lewy bodies }\end{array}$ \\
\hline Treatment & $\begin{array}{l}\text { 5-ASA (UC), steroids, thiopurine, } \\
\text { Anti-cytokine antibodies (TNF, } \\
\text { IL12/23), anti-integrin antibodies, } \\
\text { Bowel surgery }\end{array}$ & $\begin{array}{l}\text { Dopamine precursors and } \\
\text { agonists, Monoamine oxidase-B } \\
\text { inhibitors, Deep brain stimulation }\end{array}$ \\
\hline Shared features & \multicolumn{2}{|c|}{$\begin{array}{l}\text { - Common and complex chronic disease } \\
\text { - Multifactorial disorder involving genetic and environmental } \\
\text { - } \text { factors } \\
\text { - } \text { Increasing prevalence } \\
\text { - Global burden of disease } \\
\text { - Genetic pleiotropy including } L R R K 2\end{array}$} \\
\hline
\end{tabular}

CD, Crohn's disease; UC, ulcerative colitis; 5-ASA, 5-aminosalicylic acid; TNF, tumor necrosis factor; IL, interleukin; LRRK2, leucine-rich repeat kinase 2. 
Table 2. Epidemiological link between inflammatory bowel disease and Parkinson's disease based on previous population-based studies.

\begin{tabular}{|c|c|c|c|c|c|c|c|}
\hline Author & Country & Period & Number of patients & Age & ICD code & $\begin{array}{l}\text { Incidence } \\
\text { (per } 1000 \text { person- } \\
\text { years) }\end{array}$ & aHR/aIR/aOR $(95 \% \mathrm{Cl})$ \\
\hline \multicolumn{8}{|c|}{ Parkinson's disease risk among patients with inflammatory bowel disease } \\
\hline Lin et al. ${ }^{7}$ & Taiwan & $2000-2011$ & $\begin{array}{l}\text { IBD: } 8,373 \\
\text { Control: } 33,492\end{array}$ & $\geq 20$ years & ICD-9 & $\begin{array}{l}\text { IBD: } 1.7 \text {, Control: } 1.2 \\
\text { CD: } 1.8, \text { UC: } 1.3\end{array}$ & $\begin{array}{l}\text { IBD: } \text { aHR=1.4 (1.1-1.7) } \\
C D: \text { aHR=1.4 (1.1-1.8) } \\
\text { UC: aHR=0.9 (0.5-1.8) }\end{array}$ \\
\hline Peter et al. ${ }^{8}$ & USA & $2000-2016$ & $\begin{array}{l}\text { IBD: } 144,018 \\
\text { Control: } 720,090\end{array}$ & $\geq 18$ years & ICD-9 & $\begin{array}{l}\text { IBD: } 0.7 \text {, Control: } 0.6 \\
\text { CD: } 0.6, \text { UC: } 0.8\end{array}$ & $\begin{array}{l}\text { IBD: } \text { alR=1.3 (1.1-1.4) } \\
\text { CD: alR=1.3 (1.0-1.5) } \\
\text { UC: alR=1.3 (1.1-1.5) }\end{array}$ \\
\hline Weimers et al. ${ }^{9}$ & Sweden & $2002-2014$ & $\begin{array}{l}\text { IBD: } 39,652 \\
\text { Control: } 396,520\end{array}$ & $\geq 18$ years & ICD-7 ICD-10 & $\begin{array}{l}\text { IBD: } 0.4 \text {, Control: } 0.3 \\
\text { CD: } 0.3, \text { UC: } 0.4\end{array}$ & $\begin{array}{l}\text { IBD: } \text { aHR=0.9 }(0.7-1.1) \\
C D: \text { aHR }=0.9(0.6-1.4) \\
\text { UC: } a H R=0.8(0.6-1.1)\end{array}$ \\
\hline Villumsen et al. ${ }^{10}$ & Denmark & $1977-2014$ & $\begin{array}{l}\text { IBD: } 76,477 \\
\text { Control: } 7,548,259\end{array}$ & $\geq 15$ years & ICD-8, ICD-10 & IBD: 0.4 , Control: 0.2 & IBD: aHR=1.2 (1.1-1.4) \\
\hline Park et al. ${ }^{11}$ & Korea & $2010-2013$ & $\begin{array}{l}\text { IBD: } 38,861 \\
\text { Control: } 116,583\end{array}$ & All age & ICD-10 & $\begin{array}{l}\text { IBD: } 0.4 \text {, Control: } 0.2 \\
\text { CD: } 0.2 \text {, UC: } 0.6\end{array}$ & $\begin{array}{l}\text { IBD: aHR=1.9 (1.4-2.4) } \\
\text { CD: aHR=2.2 (1.1-4.5) } \\
\text { UC: aHR=1.9 (1.4-2.5) }\end{array}$ \\
\hline \multicolumn{8}{|c|}{ Inflammatory bowel disease risk among patients with Parkinson's disease } \\
\hline Camacho-Soto et al. ${ }^{13}$ & USA & 2004-2009 & $\begin{array}{l}\text { PD: } 89,790 \\
\text { Control: } 118,095\end{array}$ & $>65$ years & ICD-9 & NA & $\begin{array}{l}\text { IBD: aOR=0.9 (0.8-0.9) } \\
\text { CD: aOR=0.8 (0.7-0.9) } \\
\text { UC: aOR=0.9 (0.8-1.0) }\end{array}$ \\
\hline
\end{tabular}

ICD, international classification of diseases; aHR/aIR/aOR, adjusted hazard ratio/adjusted incidence rate/adjusted odds ratio; IBD, inflammatory bowel disease; CD, Crohn's disease; UC, ulcerative colitis; PD, Parkinson disease; NA, not applicable. 
Table 3. The pleiotropic effects of the susceptibility loci by searching for reported genetic associations of inflammatory bowel disease and Parkinson's disease by the National Human Genome Research Institute (NHGRI) GWAS Catalog ${ }^{23}$ (accessed on 22/06/2020).

\begin{tabular}{|c|c|c|c|c|c|}
\hline Locus & Disease & SNP* & Position & Reported gene(s) & $\begin{array}{l}\text { Number of previously reported SNPs in locus by GWAS } \\
\text { Catalog }\end{array}$ \\
\hline \multirow[t]{2}{*}{$1 q 22$} & IBD & rs3180018 & 155260340 & $\begin{array}{l}\text { SLC5OA1, DPM3, KRTCAP2, MUC1, MTX1, } \\
\text { SCAMP3, RUSC1, RXFP4, SSR2, EFNA1, } \\
\text { RP11-201K10.3, TRIM46, THBS3, GBA, } \\
\text { FAM189B, CLK2, HCN3, PKLR, FDPS, } \\
\text { YY1AP1, DAP3, GON4L, RIT1, KIAAOOO7, } \\
\text { UBQLN4, EFNA3, ASH1L, MSTO1, SYT11, } \\
\text { ARHGEF2 }\end{array}$ & 3 (rs4971079†, rs78973538+, rs3180018) \\
\hline & PD & rs357499011 & 155162560 & GBA & 4 (rs12726330, rs35749011, rs76763715, rs2230288) \\
\hline \multirow[t]{2}{*}{$1 \mathrm{q} 23.3$} & IBD & rs1801274 & 161509955 & HSPA6, FCGR3A, FCGR3B, FCGR2B, FCGR2A & $\begin{array}{l}5 \text { (rs10800309, rs10800314t, rs61802846†, rs1801274, } \\
\text { rs79568124†) }\end{array}$ \\
\hline & PD & rs6658353 & 161499264 & FCGR2A & 1 (rs6658353) \\
\hline \multirow[t]{2}{*}{$10 q 22.1$} & UC & rs2642575 & 69783412 & COL13A1 & 1 (rs2642575‡) \\
\hline & PD & rs17497526 & 69820364 & COL13A1 & 1 (rs17497526ł) \\
\hline \multirow[t]{2}{*}{$12 q 12$} & IBD & rs148319899 & 40346421 & SLC2A13, LRRK2, MUC19 & $\begin{array}{l}7 \text { (rs12422544, rs11175593, rs148319899, rs7313895†, } \\
\text { rs4768236, rs17466626t, rs11564258) }\end{array}$ \\
\hline & PD & rs34637584 & 40340400 & LRRK2 & 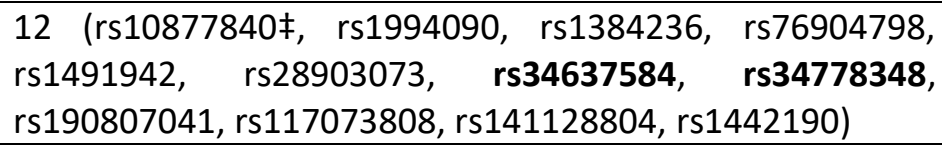 \\
\hline \multirow[t]{2}{*}{$14 q 31.3$} & IBD & rs8005161 & 88006251 & GALC, GPR65 & 3 (rs55808324, rs8005161, rs11624293†) \\
\hline & PD & rs8005172 & 88006268 & GALC, GPR65 & 2 (rs8005172, rs979812) \\
\hline \multirow[t]{2}{*}{$16 q 12.1$} & IBD & rs2066844 & 50712015 & SNX20, NOD2, CYLD, NKD1 & $\begin{array}{l}10 \quad(\mathrm{rs} 117372389 \dagger, \mathrm{rs} 2357623 \dagger, \mathrm{rs} 6596, \mathrm{rs} 17221417, \\
\text { rs2066844, rs2066845†, rs5743289, rs2076756, } \\
\left.\text { rs } 72796367 \dagger, \mathrm{rs} 2066847 / \mathrm{rs} 5743293^{\S}\right)\end{array}$ \\
\hline & PD & rs6500328 & 50702745 & NOD2 & $1(\mathrm{rs6500328)}$ \\
\hline
\end{tabular}

*The most significant SNP within each locus from the largest study. +SNPs reported in the cross-phenotype meta-analysis of immune diseases. $¥$ SNPs reported in the GWAS Catalog ${ }^{23}$ but not reaching genome-wide significance level (P-value is between $5 \times 10^{-6}$ and $\left.6 \times 10^{-7}\right)$. ${ }^{8}$ rs5743293 has merged into rs2066847. Missense/frameshift variants are marked in bold.

SNP, single nucleotide polymorphism; IBD, inflammatory bowel disease; PD, Parkinson disease; UC, ulcerative colitis. 


\section{Figure Legends}

Figure 1. Publication trends on PubMed (1971-2019). Number of publications in PubMed per year using keywords [((Crohn's disease) OR ulcerative colitis) OR inflammatory bowel disease) AND Parkinson's disease]. The number of publications in this area of research is increasing rapidly. 
Figure 2. Possible connections between inflammatory bowel disease (IBD) and Parkinson's disease (PD). 
Figure 3. LRRK2 variants and their association with inflammatory bowel disease and/or Parkinson's disease. Variants associated with inflammatory bowel disease are marked with the bowel symbol, those associated with Parkinson's disease with the brain symbol. A red symbol means increased disease risk, blue means decreased disease risk. Consequences of the functional variants with pleiotropic effects for LRRK2 kinase and GTPase activity, and for LRRK2 state is indicated in the table. CD, Crohn's disease; PD, Parkinson disease. 
Figure 4. Schematic overview of the gut-brain axis and how gut inflammation could contribute to Parkinson's disease. (A) The gut-brain axis provides bidirectional communication between the gut microenvironment and the central nervous system, via projections of the vagal nerve and the peripheral immune system. Gut-derived signals generated by diet and gut microbiota are known to affect behaviour and mood. Vice versa, emotional stress and psychological conditions can influence the gut. (B) When gut inflammation is uncontrolled it can lead to abnormal $\alpha$-synuclein deposition in the gut, and subsequent spread of misfolded $\alpha$-synuclein to the brain. Thus, effective treatment of inflammatory bowel disease (IBD) might modify the risk of developing Parkinson's disease (PD). 